\title{
Context based GIS Location Information System
}

\author{
G. K. Mohan Devarakonda \\ Research Scholar \\ Department of Computer Science \\ Krishna University
}

\author{
Y. K. Sundara Krishna, PhD \\ Professor \\ Dept. of Computer Science \\ Krishna University
}

\begin{abstract}
The current trend in mobile computing is revolving around the context of the user. These mobile computing services start their applications by first determining the context of the user, and then provide the service to the user accordingly. So far, the service providers of the said services are using the latitude and longitudinal measures to identify the Geographical location of the user. This paper surveys various location based attributes that can also be used to identify the context of the user such as Geographical location, Height with respect to sea level, etc..,
\end{abstract}

\section{Keywords}

Geographical Information Systems, Context based services, location aware services, attributes

\section{INTRODUCTION}

The mobile computing services of different dimensions can be provided now a day's based on the context of the user. For an example, E-Commerce related services such as Flipkart, Snapdeal are first identifying the location of the user to determine whether the user is eligible to provide their services because the said E-commerce services provide their services only to certain areas.

On the other side, these service providers are using the geographical location measures such as latitude and longitude to identify the location of user. In this paper, we identified several common attributes that are used to identify the context of the user and its related attributes.

\section{GEOGRAPHICAL INFORMATION SYSTEM (G.I.S)}

G.I.S is used to analyze the things and events that happen on earth with the use of spatial data such as latitude, longitude or any national grid coordinate for explicit reference. It is a system which is composed of five different working components namely Hardware, Software, Data, People and Methods.

G.I.S maintains information as a collection of layers that can be linked together by geography and includes data such as address, postal code, and road name for implicit reference. A process called geo-coding is used to create explicit references from implicit references.

A general purpose G.I.S essentially performs five tasks ${ }^{[1]}$

1. Data Acquisition (Digitization)

2. Data Manipulation

3. Data Management

4. Query and Analysis

5. Output Visualization
The technologies that are closely related to the geographical information system include CAD, Desktop Mapping, Remote sensing and GPS, DBMS. Along with the above technologies, the system also provides various sophisticated tools to store, analyze and display geographical information.

G.I.S systems work with two different types of geographical models namely Vector and Raster ${ }^{[1]}$. In the vector model, information about points, lines and polygons is encoded and stored as a collection of $\mathrm{x}, \mathrm{y}$ coordinates. Thus the location of a point feature is described by a single $\mathrm{x}, \mathrm{y}$ coordinate. The vector model is best suitable for describing discrete features. The Raster model is evolved to model continuously varying features such as soil type. Modern G.I.S systems are able to handle both vector and raster models.

Data in many different forms can be entered into GIS ${ }^{[2]}$. Data that are already in map form can be included in GIS. This includes such information as the location of rivers and roads, hills and valleys. Digital, or computerized, data can also be entered into GIS. An example of this kind of information is data collected by satellites that show land use - the location of farms, towns, or forests. GIS can also include data in table form, such as population information. GIS technology allows all these different types of information, no matter their source or original format, to be overlaid on top of one another on a single map.

People working in many different fields use GIS technology. Many businesses use GIS to help them determine where to locate a new store. Biologists use GIS to track animal migration patterns. City officials use GIS to help plan their response in the case of a natural disaster such as an earthquake or hurricane. GIS maps can show these officials what neighborhoods are most in danger, where to locate shelters, and what routes people should take to reach safety. Scientists use GIS to compare population growth to resources such as drinking water, or to try to determine a region's future needs for public services like parking, roads, and electricity. There is no limit to the kind of information that can be analyzed using GIS technology. With the help of GIS, people can compare the locations of different things in order to discover how they relate to each other.

\section{CONTEXT AWARENESS}

A property of mobile device that is defined complimentarily to the location is called context. Context Awareness is a term which sought to deal with linking changes in environment with computer systems. Particularly in computer science, context awareness refers to the idea that computers can sense and react based on their environment. These devices may have information about the circumstances under which they are able to operate and react accordingly. 
Context aware systems are concerned with the acquisition of context, the abstraction and understanding of context. As the user's activity and location are crucial for many applications, context awareness has been focused more deeply in the research fields of location awareness and activity recognition.

Context Awareness is used to design innovative interfaces and is often used as a part of ubiquitous and wearable computing ${ }^{[3]}$. Context Awareness has its applications in health care, industrial production, pervasive games etc.,

Context encompasses more than just the user's location, because other things of interest are also mobile and changing. Context includes lighting, noise level, network connectivity, communication costs, communication bandwidth, and even the social situation ${ }^{[4]}$.

If the geographical information is integrated with context awareness more services will be provided to the users based on the user's context ${ }^{[4]}$.

\section{PROPOSED CONTEXT ATTRIBUTES}

The context of a user is identified now a day by means of latitude and longitude which represents the geographical location of the user. In addition to these two measures, the context can also be identified by means of various attributes include the following : Height with respect to sea level, height with respect to ground level, Temperature, Humidity, Area type, Business Type, Status of the object and Object Type. The attributes which are specified in the list above can also be used to identify the context of a user and can help even to identify several other related data.

\subsection{Latitude \& Longitude}

These attributes are used to identify the exact geographical location of the user/object. The latitude and longitude are the measures in the form of meridians. The latitude of a point $p$ on a surface is defined as the angle that a straight line passing through both $\mathrm{p}$ and Center $\mathrm{C}$ subtends with respect to the equatorial plane. Latitude angles can range up to $+90^{\circ} \mathrm{N}$ and $-90^{\circ} \mathrm{S}$. The longitude angle can range up to $+180^{\circ} \mathrm{E}$ and Down to $-180^{\circ} \mathrm{W}$.

\subsection{Height}

The height of an object is defined as the vertical distance from base to top measured along the vertical axis between a specified point and another usually in meters globally with respect to sea level and locally with respect to ground level. Height has its importance in the construction fields to provide a good sense of spatial enclosure. For example, If the height of buildings along a street is too low relative to the street width, the space will evoke only a weak sense of spatial enclosure and will not feel particularly inviting.

\subsection{Temperature}

The temperature of an object also plays a vital role in determining the context of the object whose range varies in between absolute zero $\mathrm{K}\left(-273.15^{\circ} \mathrm{c}\right)$ and 3.6 billion degrees centigrade. As every living object maintains homeostasis, we can check whether the temperature is within the prescribed limits and suggest needful guidelines or provide the necessary service.

\subsection{Humidity}

Humidity is defined as the amount of water vapor in air. It will be measured as Absolute Humidity, Specific humidity

and Relative Humidity. Absolute humidity is the measure of amount of water vapor in air; Specific humidity is the ratio of water to air on a mass basis while the relative humidity is the ratio of current humidity to the maximum possible at a given temperature. These measures help identify the location in a variety of ways. As Relative humidity changes with respect to temperature, sometimes it is observable to detect humidity where there are limited chances of measuring the temperature.

\subsection{Area Type}

The possible values of this attribute may be Residential, Industrial, Jungle, hill, Sea etc., which represents a variety of context locations.

\subsection{Business Type, Status}

There are several possible values for the business type and status attributes. Business type may be a Hotel, Railway etc., and status may be Dangerous, Non Dangerous, Prohibited area etc.,

\subsection{Object Type}

The objects may be defined as four types in general which include Static living objects, Dynamic living objects, Static Non-living objects and Dynamic Non-living objects. Area type, business type and object type attributes are useful where there is need of tracking, authentication and authorization services $^{[2]}$.

\section{PROPOSED ARCHITECTURE} Fig. Context Based G.I.S System Architecture

$$
\text { Context Based G.I.S Applications }
$$

Context aware Query Processing System

\begin{tabular}{|c|c|c|}
\hline \multicolumn{3}{|c|}{ RDBMS } \\
\hline \multirow{5}{*}{$\begin{array}{c}\text { G.I.S } \\
\text { Attributes(static) : } \\
\text { Latitude } \\
\text { Longitude }\end{array}$} & \multicolumn{2}{|c|}{ Context Based System } \\
\hline & Static info & $\begin{array}{c}\text { Dynamic } \\
\text { info }\end{array}$ \\
\hline & Height & Temperature \\
\hline & Land Type & Humidity \\
\hline & & Pressure \\
\hline
\end{tabular}

The proposed system works by identifying the location of the user using G.I.S attributes and Static, Dynamic contexts of the location. Based on the attributes identified, context based RDBMS will be ready which will serve as a base for the entire system. This RDBMS should be able to map the context of user with the location context to provide effective services. User can now be able to interact with the system through the context aware query processing system and can avail a variety of services based on his requirement.

\section{ADVANTAGES}

a) More services may be provided to the user in case of emergency or any other situations.

b) Helpful in getting some more information about the context of the user/object. 
c) Users can get services according to their requirements.

d) More useful for users with smart phone and touch based devices.

\section{DISADVANTAGES}

a) May not be appropriate in some situations as context cannot be identified in every situation.

b) May not be useful for all types of devices

c) Device compatibility issues may arise.

\section{CONCLUSION}

Defining or identifying the context of a location or user using the above said attributes may be useful in collecting some more context related information and can be helpful in providing new and effective services to the users in a variety of ways. One can think of using the above said attributes for some more productivity to the services which are provided on the base of context.

Mapping the choice of the user (user context) with the location with the use of a context aware DBMS enables the user to search for the data of his own requirement with more clarity.
For example, a user who is in search of a restaurant may opt his choice to be vegetarian (user context) and the GIS attributes of the user location (location context) already maintains the restaurants list of the location. Now by integrating (mapping) both the contexts, the context aware query processing system filters out all vegetarian restaurants nearby. Hence, it is easy for the user to find out the right choice for his dinner.

This concept may be extended to various user contexts including educational context, Traffic context, weather context etc.

\section{REFERENCES}

[1] http://gisgeography.com

[2] "Principles GIS", Otto Huisman and Rolf A.de By

[3] "A Context-Aware Preference Database System" Kostas Stefanidis , Evaggelia Pitoura, Panos Vassiliadis, 2005.

[4] "Context Aware Computing Applications", Bill N. Schilit, Norman Adams, and Roy Want 